\title{
EFFECTS OF TOBACCO USE ON ORAL HEALTH - AN OVERVIEW
}

K.H. Awan. Effects of tobacco use on oral health - an overview. Annal Dent Univ Malaya 2011; 18: 18-23.

\section{ABSTRACT}

Tobacco use is linked with many serious illnesses, such as cancer, cardiopulmonary diseases, as well as with many health problems. Every year, the use of tobacco products causes a heavy toll of deaths and severe human disease worldwide. One of the many health problems linked to tobacco use is its detrimental impact on oral health. Tobacco causes a whole series of oral health problems, ranging from life-threatening (precancerous changes leading to oral cancer) and serious (periodontal disease, teeth decay) to social (bad breath). Tobacco is consumed through the mouth in a variety of forms, varied from smoked tobacco to smokeless tobacco chewing on itself or combined with areca nut. All these forms of tobacco have damaging effects on the oral health. The most significant preventive measure to prevent the oral health problems caused by tobacco use is to stop using tobacco products. The risk of developing oral cancer drops rapidly when a smoker ceases tobacco use. After ten years of not using tobacco, an ex-smoker/user's risk of oral cancers is about the same as that for someone who has never smoked. To stop using tobacco products is not an easy task. Fortunately, there are a number of therapies available to assist in quitting of tobacco. It is important to remember that, while it will be difficult, ceasing to use tobacco has immediate health benefits, including increased life expectancy and reduced risk of tobacco related diseases and conditions.

Key words: Tobacco, smoking, Oral cancer, betel quid, prevention

\section{INTRODUCTION}

Tobacco is the single greatest cause of preventable death globally (1). Tobacco is consumed in a variety of different ways, though smoking of manufactured cigarettes is the most prevalent form of its use. The emergence of widespread cigar use particularly among adolescents of both sexes has been reported in the past decade in the US. Cigars have higher total nicotine content than cigarettes do and can deliver nicotine both through smoke and through direct oral contact with the tobacco wrapper. Cheroots are small cigars made of heavy bodied tobacco. Bidi smoking is a popular form of tobacco use in south Asia, accounting for one-third of the tobacco produced in India for smoking. Bidis and Kreteks are gaining popularity
Review Article

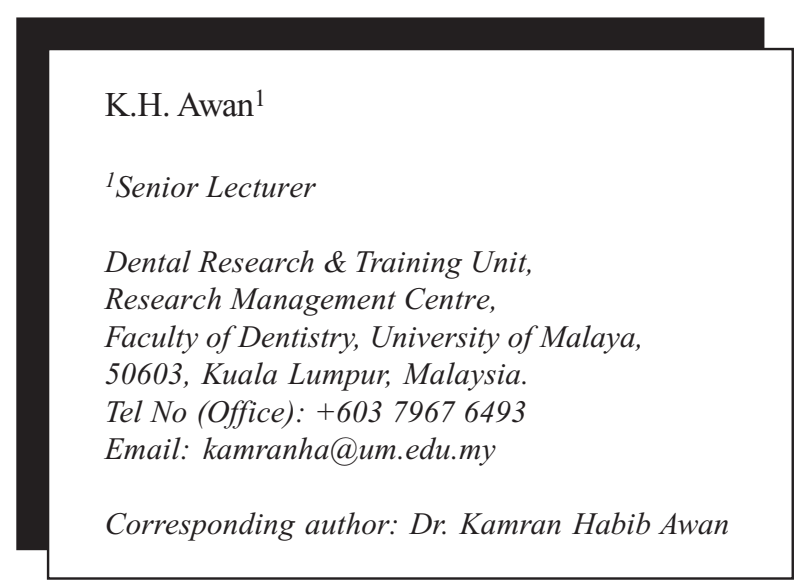

among young people in North America, and more than $15 \%$ of adolescent smokers use these tobacco products. Pipe smoking is one of the oldest methods of smoking and was brought to Europe by sailors from America. Water pipes include special receptacles through which smoke has to pass, ostensibly to reduce its harmful effects. Hookah is an Indian water pipe. The habit of reverse smoking by holding the glowing end of cigarettes or cigars within the oral cavity is described in parts of India, South America and the Philippines. The habit is practiced extensively by older women living in rural areas.

\section{Smoked tobacco}

Many toxins are present in tobacco smoke. Tobacco smoke is made up of "side-stream smoke" from the burning tip of the cigarette and "main-stream smoke" from the filter or mouth end. Tobacco smoke contains thousands of different chemicals which are released as particles and gases. The particulate phase includes nicotine, "tar" (itself composed of many chemicals), benzene and benzo(a)pyrene. The gas phase includes carbon monoxide, ammonia, dimethylnitrosamine, formaldehyde, hydrogen cyanide and acrolein. Some of these have marked irritant properties and some 60, including benzo(a)pyrene and dimethylnitrosamine, have been shown to cause cancer.

The tar yield of different brands of cigarettes range from $0.5 \mathrm{mg}$ to $26 \mathrm{mg}$ (averaging $12.5 \mathrm{mg}$ ), with the most popular brands containing $15-17 \mathrm{mg}$ of tar. In the European Union (EU) cigarettes have to contain less than $12 \mathrm{mg}$ of tar from 1998 (2). Nicotine yields range from $0.05 \mathrm{mg}$ to $1.7 \mathrm{mg}$, with the most popular brands yielding $1.0 \mathrm{mg}$ of nicotine. In developed countries over $95 \%$ of manufactured cigarettes consumed are filter-tipped.

About $10 \%$ consume tobacco as roll-your-own cigarettes mostly among lower socioeconomic groups. 


\section{Smokeless (chewing) tobacco (ST)}

There are two main types of smokeless tobacco chewing tobacco and snuff. The most written about is smokeless tobacco use among Asians taken with betel/ areca quid. Over 90 percent of Indians add tobacco to the betel quid mixture. Commercially prepared betel quid products that contain mostly areca nut and flakes of tobacco are called Gutka. Other ST products which carry significant mutagenicity are Toombak used in the Sudan, Shamma in the Jizan province in Saudi Arabia, powdered tobacco and alkali mixtures such as Nass/ Naswar used in northern and central Asia and in Pakistan, Khaini (a mixture of ST and lime) used in Bihar state of India and Nepal, and boiled/sweetened ST called Zarda mostly used by people from Bangladesh (3). All these forms of tobacco use are associated with an increased risk of oral cancer.

\section{Second-hand or environmental tobacco smoke (ETS)}

ETS is carcinogenic to human beings. Metaanalyses have shown a significant association between lung cancer and smoke exposure from a spouse and also between lung cancer and exposure at work. Risks for other cancer types are inconclusive. There is at present insufficient evidence that children exposed to parental smoke have an altered risk of developing any cancer.

\section{Epidemiology}

Tobacco which annually kills 4.9 million people worldwide at present is estimated to take 10 million lives every year by 2020 . The more depressing part is that half of them will die in their middle age. A global estimate of smoking prevalence by each country is given in a WHO data base for reference (4). These are based on adult and youth smoking behaviours collected from population-based, cross sectional surveys at a given point of time. China is the largest producer of tobacco in the world as well as the largest consumer. A national prevalence survey in 1996 among adults (age 15+) found that $63 \%$ of males smoked. In Malaysia, three National Health and Morbidity surveys which have been conducted since 1986, the prevalence of smoking among adults age 18 and above were more than $20 \%$; i.e. $21.5 \%$ in 1986 , $24.8 \%$ in 1996 and $22.8 \%$ in $2006(5,6)$.

National surveys of persons aged 18 and older from 1970 onwards report a decline in prevalence in USA and most western European countries $(7,8)$. The rate of decline among women is less than for men, and the quit index for men is substantially higher than for women. Both in UK and USA there are now twice as many former cigarette smokers as current smokers. In general, the prevalence of smoking in most population groups is lowest among those with the highest educational level. By race, smoking among adult blacks is similar to whites in most countries. Denmark has the highest rates of smoking in the EU.

\section{Clinical presentation}

\section{General effects}

Smoking-attributed diseases include cancer of trachea, lung, bronchus, lip, mouth and pharynx, ischemic heart disease, stroke, hypertension, bronchitis, chronic obstructive pulmonary disease, emphysema and asthma. Due to substantial decrease in smoking in countries such as the UK, male lung cancer rates have decreased rapidly in the last decade (9). On current trends, the annual number of smokingattributable deaths among women should exceed that for men shortly after the year 2000 .

\section{Effects of tobacco on teeth and oral health}

The damaging and harmful effects of tobacco usage on oral health are now well recognized, in particular a higher prevalence and severity of periodontal diseases among smokers and the association of tobacco use with candidosis $(10,11)$, and with oral malignancies $(12,13)$. Several recent documents have reviewed the scientific evidence relating to the oral disease burden attributable to tobacco use (14) and have highlighted the role and the need for the dental profession to get involved with tobacco intervention (15).

Smoking cause's discolouration of teeth and some argue that tobacco in fact might increase dental decay as it lowers salivary $\mathrm{pH}$ and the buffering power. Smoking is likely to cause halitosis and may affect smell and taste. Smokers may present with generalised melanosis of the oral mucosa that often necessitate investigations to exclude other systemic disorders. Wound healing is impaired in tobacco smokers possibly due to local vasoconstriction and poor neutrophil function. There is fair evidence that tobacco use is a major factor in the progression of periodontal disease (16-20). Smokers have an increased prevalence of periodontitis, and their disease severity is higher with greater alveolar bone loss resulting in deeper pockets compared with non-smokers (11). Acute necrotising ulcerative gingivitis (ANUG) has been shown to be associated with heavy smoking. Periodontal therapy often fails among smokers and it is difficult to halt attachment loss. Possibly for similar reasons dental implant failure is more common in smoking subjects compared with non-smokers.

\section{Oral cancer}

Over 80 percent of oral cancers are associated with tobacco use (21). Oral squamous cell carcinoma presents in a variety of ways such as white and red patches, non-healing ulcers or exophytic growths. Most early lesions are asymptomatic. Persistent ulceration with rolled margins and fixation to underlying tissues are pathognomonic signs of oral malignancy. In late stages, disease spreads to adjacent structures notably involving regional lymph nodes, and 
can cause mobile teeth and loss of teeth or even pathological mandibular fractures. These stages may be associated with pain, numbness or paraesthesia. The clinical features of oral cancer are described elsewhere. Currently diagnosing oral cancer relies on pathological examination by biopsy and use of imaging techniques to estimate the spread of the disease. In addition, a number of adjunctive tools are available which may facilitate the diagnosis of oral cancer in the early stages (22-24).

\section{Oral leukoplakia}

Oral leukoplakia is the most common potentially malignant lesion defined as a predominantly white lesion of oral mucosa that cannot be characterised as any other definable lesion (25). The appearance of leukoplakia varies from uniformly white homogeneous lesions to non-homogeneous speckled lesions with red and/or nodular features. Leukoplakia is often associated with tobacco use though idiopathic forms of leukoplakia are recognised. The site of the oral cavity affected by leukoplakia is often said to be associated with the type of tobacco habit practiced; lateral tongue and floor of mouth in cigarette smokers, palate in pipe smokers and reverse smokers, commissures in bidi smokers, buccal groves in tobacco chewers where they park the quid and lower or upper labial mucosa in snuff dippers. In a recent study in the Netherlands, $64 \%$ of men and $60 \%$ of women with oral leukoplakia were smokers (26). Tobacco use in men was significantly associated with leukoplakia of buccal mucosa and with all leukoplakia of floor of mouth in both sexes. Oral leukoplakia in smokers needs to be investigated by biopsy to assess any dysplasia. Moderate to severe oral epithelial dysplasia when present necessitate surgical intervention. Intervention studies have demonstrated that leukoplakia present in smokers might be reversible when smoking habit was reduced or given up.

\section{Erythroplakia}

Erythroplakia is defined as 'A fiery red patch that cannot be characterized clinically or pathologically as any other definable disease' (27). Tobacco may underlie some cases of erythroplakia.

\section{Smoker's palate (Leukokeratosis nicotina palati)}

A greyish white discolouration of the palate with multiple red elevated dots (inflamed minor salivary gland openings) is often encountered in chronic smokers. This is considered as a benign lesion as cancer is not known to arise from this benign keratosis.

\section{Reverse smoker's keratosis}

This is serious potentially malignant lesion encountered in people who place the glowing end of the cigar or cigarette inside the mouth. The clinical appearance is often a mixture of red and white plaques. Excrescences are found within the lesion corresponding to inflamed minor salivary glands. Whereas aforementioned smoker's palate is not considered as a precancerous lesion, palatal changes in reverse smokers are a high risk lesion that is associated with cancer development (28).

\section{Aetiopathogenesis}

Tobacco smoking (i.e., cigarette, pipe or cigar smoking) particularly when combined with heavy alcohol consumption has been identified as the primary risk factor for approximately $80 \%$ of oral malignancies (29). The risk of oral and pharyngeal cancers is similar for cigarette and cigar smokers, with an overall risk seven to ten times higher than for never smokers. This is not surprising as the oral cavity is exposed to the carcinogens in smoke whether the smoke is inhaled or not. When the frequency of daily tobacco use is computed, there is strong dose response relationship between smoking rates and risk of mouth cancer. Addition of ST to the areca quid raises the relative risk of the product by nearly 15 times.

There are 55 carcinogens (Table 1) in cigarette smoke that have been evaluated by the International Agency for Research on Cancer (IARC) and for which there is "sufficient evidence for carcinogenicity" in either laboratory animals or humans (30). Other carcinogens not evaluated by the IARC may also be present.

Table 1. Summary of carcinogens in tobacco*

\begin{tabular}{lc}
\hline Type & $\begin{array}{c}\text { No. of } \\
\text { compounds }\end{array}$ \\
\hline Polycyclic aromatic hydrocarbons (PAH) & 10 \\
N-Nitrosamines & 7 \\
Aza-arenes & 3 \\
Aromatic amines & 3 \\
Heterocyclic aromatic amines & 8 \\
Aldehydes & 2 \\
Miscellaneous organic compounds & 15 \\
Inorganic compounds & 7 \\
\hline
\end{tabular}

*According to the International Agency For Research on Cancer (IARC), http://www.iarc.fr

Case-control studies from Europe have reported adjusted odds ratios (ORs) of 11.1 for oral cavity and 12.9 for pharyngeal cancer (31). In particular, smoking frequency and duration, and age at start have significant associations. After giving up tobacco for a decade or so, the risk of oral cancer of a past smoker drops significantly to levels almost comparable to never smokers.

Smoking patients show reduction of inflammatory clinical signs that might be associated with local vasoconstriction from nicotine, influence on vasculature and cellular metabolism. This may suppress symptoms of gingival inflammation. Pathogenesis of periodontitis in smokers could be linked to defects in neutrophil function, impaired 
serum antibody responses to periodontal pathogens and potentially diminished gingival fibroblast function suggesting altered host response and susceptibility (32). It is claimed that among smokers, more patients remain culture positive for periodontal pathogens after therapy. This may contribute to the often observed unfavourable treatment results among non-compliant smokers.

\section{Diagnosis}

Detection of tobacco consumption is mostly based on taking a social history. This should include questions on type of tobacco habit, daily frequency and duration of use. Age of commencement is also an important risk factor for many disorders and should be recorded. Current smokers could be regular or occasional smokers, regular being daily smokers. Some have the habit of binge smoking when consuming alcohol only but are unlikely to be addicted to tobacco.

Tobacco handling can usually be seen on heavily smoking patients' fingers and the tobacco stains on the oral mucosa and teeth. Dorsal tongue is often stained in many smokers. A bad breath can also highlight a smoker.

Validation of smoking can be done using the carbon monoxide breath test (piCO, Bedfont) or by measuring salivary, urine or serum cotinine which is a metabolite of nicotine. Cut off concentration of salivary cotinine is taken as $14 \mathrm{ng} / \mathrm{ml}$ to detect a regular smoker.

Level of dependence to tobacco can be assessed using the Fagerström test.

\section{Treatment and interventions}

Tobacco dependence shows many features of a chronic disease. Regular smokers are addicted to the habit as tobacco use results in true drug dependence. A minority is able to quit in one attempt but the majority may need some assistance to cease tobacco use. Numerous effective treatments are now available, and the dentists, oral physicians and their team members should become actively involved in efforts to reduce smoking.

Smoking cessation advices delivered by dentists have shown to be effective. Brief advice given by a clinician lasting about 3 minutes can yield a cessation rate up to $5 \%(33,34)$. With additional support such as recommended use of nicotine replacement therapy the quit rates achieved could be doubled. In treating a smoker (willing to quit) the 5A's (35), designed as a brief counselling intervention, is helpful (Table 2).

\section{Pharmacotherapy}

As with other chronic diseases, the most effective treatment of smoking requires multiple approaches in addition to clinician's advice. Pharmacotherapy is proven to be effective, and several products are available; nicotine patches, nicotine gum, nicotine lozenge, nicotine inhaler and nicotine nasal spray.
Table 2. The 5 A's of tobacco cessation*

1. Ask about tobacco use - every patient/every visit

2. Assess willingness to make a quit attempt

3. Advice (those willing) to quit tobacco use. Those unwilling will need motivation to return to the topic at a later time

4. Assist in quit attempt - set a quit date, emphasize total abstinence, prompt support seeking, provide supplementary material and recommend pharmacotherapy (see below)

5. Arrange follow up and refer to a specialist clinic if the quit attempt has failed

* Fiore MC. US public health service clinical practice guideline: treating tobacco use and dependence. Respir Care. 2000; 45(10): 1200-62.

Non-nicotine medication approved for smoking cessation is bupropion (Zyban) therapy starting one week before the quit date and continued up to 12 weeks after quitting. There are several medical contraindications particularly those with a predisposition to seizures and/or a history of epilepsy. Pregnant and breast-feeding mothers should not be prescribed this drug. Bupropion therapy increases the chances of quitting considerably up to $30 \%$.

\section{Prevention}

Prevention of tobacco use is a key element in public health. As tobacco use and experimentation starts in early life preventive approaches should be appropriately targeted to young people. Paedodontists, orthodontists, school dentists and family practitioners can take steps to initiate advice to young children never to start smoking. Banning tobacco smoking in public places, legislation on tobacco advertising and taxation are known to effect tobacco sales. Primary prevention, the helping of people not to use tobacco in the first place and assisting current smokers to quit, is an effective way to reduce morbidity and mortality from oral cancer.

\section{CONCLUSION}

The oral health ill-effects of tobacco in whole or in part are well known, and there is weighty evidence that tobacco use has considerable influence on oral health. But tobacco use is a modifiable risk factor for oral diseases, and an obvious professional interest in tobacco intervention can make a big difference in the health of an individual or the outcome of a given disease. Dentists have probably the greatest access to 'healthy' tobacco users in the healthcare system, and even in the absence of tobacco-related diseases in the mouth, the dentist will easily recognize these patients. Therefore, dentists should pursue more formal training in tobacco cessation counselling, which should be as much a part of their job as plaque control and dietary advice. 


\section{REFERENCES}

1. WHO Report on the Global Tobacco Epidemic, 2008: the MPOWER package. Geneva: World Health Organization. 2008. ISBN 978-92-4159628-2.

2. http://eur-lex.europa.eu/LexUriServ/LexUriServ. do?uri=CELEX:32001L0037:en:NOT

3. International Agency for Research on Cancer (IARC). Monographs on the Evaluation of the Carcinogenic Risk of Chemicals to Humans, Vol. 89. Smokeless Tobacco and Some Tobaccospecific N-Nitrosamines. Lyon: IARC; 2007.

4. World Health Organization. Addressing the Worldwide Tobacco Epidemic through Effective Evidence-Based Treatment. Expert Meeting March 1999, Rochester, Minnesota, USA. Tobacco Free Initiative, WHO 2000.

5. Public Health Institute Ministry of Health Malaysia. National Health and Morbidity Survey, 1986-1987, Volume IV- Cigarette Smoking.

6. Public Health Institute Ministry of Health Malaysia 2008. The Third National Health and Morbidity Survey-Smoking, 2006.

7. Wald N, and Nicolaides-Bouman A. UK Smoking Statistics. 1991: OUP.

8. General Lifestyle Survey: Smoking and drinking among adults, 2009, Office for National Statistics. 2011.

9. Quinn M, Cooper N, Rowan S. Cancer Atlas of the United Kingdom and Ireland 1991-2000. Office for National Statistics, 2005.

10. Bergstrom J, Eliasson S, Dock J. 10-year prospective study of tobacco smoking and periodontal health. J Periodontol 2000, 71: 133847.

11. Tomar SL, Asma S. Smoking-attributable periodontitis in the United States: findings from NHANES III. J Periodontol 2000, 71: 743-51.

12. Johnson NW. Tobacco use and oral cancer: a global perspective. J Dent Educ 2001, 65: 328-39.

13. Winn DM. Tobacco use and oral disease. J Dent Educ 2001, 65: 306-12.

14. EU Working Group on Tobacco and Oral Health. Meeting report. Oral Dis 1998, 4: 484-67.
15. Gordon JS, Severson HH. Tobacco cessation through dental office settings. J Dent Educ 2001, 65: 354-63.

16. Grossi SG, Zambon JJ, Ho AW, Koch G, Dunford RG, Machtei EE, Norderyd OM, Genco RJ. Assessment of risk for periodontal disease. I. Risk indicators for attachment loss. Journal of Periodontology 1994, 65: 260-267.

17. Magnusson I, Walker CB. Refractory periodontitis or recurrence of disease. Journal of Clinical Periodontology 1996, 23: 289-292.

18. Hildebolt CF, Pilgram TK, Dotson M, YokoyamaCrothers N, Muckerman J, Hauser J, Cohen S, Kardaris E, Vannier MW, Hanes P, Shrout MK, Civitelli R. Attachment loss with postmenopausal age and smoking. Journal of Periodontal Research 1997, 32: 619-625.

19. Page RC, Beck JD. Risk assessment for periodontal diseases. International Dental Journal 1997, 47: 61-87.

20. Norderyd O, Hugoson A. Risk of severe periodontal disease in a Swedish adult population A cross sectional study. Journal of Clinical Periodontology 1998, 28: 1022-1028.

21. Warnakulasuriya S, Sutherland G, Scully C. Tobacco, oral cancer and treatment of dependence. Oral Oncol 2005, 41(3): p. 244-260.

22. Awan KH, Yang YH, Morgan PR, Warnakulasuriya S. Utility of toluidine blue as a diagnostic adjunct in the detection of potentially malignant disorders of the oral cavity - a clinical and histological assessment - Oral Disease 2012 doi:10.1111/ j.1601-0825.2012.01935.x

23. Awan KH, Morgan PR, Warnakulasuriya S. Utility of chemiluminescence (ViziLite) in the detection of oral potentially malignant disorders and benign keratoses. J Oral Pathol Med 2011, 40(7): 541-4.

24. Awan KH, Morgan PR, Warnakulasuriya S. Evaluation of an autofluorescence based imaging system (VELscope) in the detection of oral potentially malignant disorders and benign keratoses. Oral Oncol 2011, 47(4): 274-7.

25. Warnakulasuriya S, Johnson NW, van der Waal I. Nomenclature and classification of potentially malignant disorders of the oral mucosa. J Oral Pathol Med 2007, 36: 575-580. 
26. Schepman KP, Bezemer PD, van der Meij EH, Smeele LE, van der Waal I. Tobacco usage in relation to the anatomical site of oral leukoplakia. Oral Dis 2001, 7: 25-27.

27. Pindborg JJ, Reichart PA, Smith CJ, Van der Waal I. World Health Organization International Histological Classification of Tumours. Histological typing of cancer and precancer of the oral mucosa. Berlin: Springer, 1997.

28. Alvarez Gómez GJ, Alvarez Martínez E, Jiménez Gómez R, Mosquera Silva Y, Gaviria Núñez AM, Garcés Agudelo A, Alonso Duque A, ZabalaCastaño A, Echeverri González E, Isaac Millán M, Ramírez Ossa D. Reverse smokers's and changes in oral mucosa. Department of Sucre, Colombia. Med Oral Patol Oral Cir Bucal 2008 Jan, 13(1): E1-8.

29. Johnson NW, Jayasekara P, Amarasinghe AA. Squamous cell carcinoma and precursor lesions of the oral cavity: epidemiology and aetiology. Periodontol 2000-2011, 57: 19-37.
30. Hoffmann D, Hoffmann I. The changing cigarette, 1950-1995. J Toxicol Environ Health 1997, 50: 307-64.

31. EU Working Group on Tobacco and Oral Health. Meeting Report. Oral Dis 1998, 4: 464-467.

32. Mullally BH. The influence of tobacco smoking on the onset of periodontitis in young persons. TobInduc Dis 2004, 2(2): 53-65.

33. Warnakulasuriya S. Effectiveness of tobacco counselling in the dental office. J Dent Education 2002, 66: 1079-1087.

34. Smith SE, Warnakulasuriya KAAS, Feyeraband C, Belcher M, Cooper DJ, Johnson NW. A smoking cessation programme conducted through dental practices in the UK. Br Dent J 1998, 185: 299-303.

35. Fiore MC. US public health service clinical practice guideline: treating tobacco use and dependence. Respir Care 2000, 45(10): 1200-62. 